\title{
Supersymmetry breaking in the three-dimensional nonlinear sigma model
}

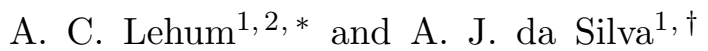 \\ ${ }^{1}$ Instituto de Fúsica, Universidade de São Paulo \\ Caixa Postal 66318, 05315-970, São Paulo, São Paulo, Brazil \\ ${ }^{2}$ Escola de Ciências e Tecnologia, Universidade Federal do Rio Grande do Norte \\ Caixa Postal 1524, 59072-970, Natal, Rio Grande do Norte, Brazil
}

In this work we discuss the phase structure of a deformed $\mathcal{N}=1$ supersymmetric nonlinear sigma model in a three-dimensional space-time. The deformation is introduced by a term that breaks supersymmetry explicitly, through imposing a slightly different constraint to the fundamental superfields of the model. Using the tadpole method, we compute the effective potential at leading order in $1 / N$ expansion. From the gap equations, i.e., conditions that minimize the effective potential, we observe that this model presents two phases as the ordinary model, with two remarkable differences: 1) the fundamental fermionic field becomes massive in both phases of the model, which is closely related to the supersymmetry breaking term; 2) the $O(N)$ symmetric phase presents a meta-stable vacuum.

PACS numbers: 11.30.Pb,11.30.Qc

* andrelehum@ect.ufrn.br

$\dagger$ ajsilva@fma.if.usp.br 


\section{INTRODUCTION}

The Nonlinear Sigma model (NLSM) was first proposed to investigate the interaction between pions and nucleons [1]. In lower dimensional systems, it is used to describe several aspects of condensed matter physics, for example, applications to ferromagnets [2 [5]. In addition, this model provides a very good theoretical laboratory containing an interesting phase structure and at same time shares with the wealth of more realistic theories, being a simple example of an asymptotically free theory [6, 7]. Recently, was conjectured that the $O(6)$ Sigma model emerges as a scaling function in AdS/CFT correspondence [8, 9].

The $O(N)$ NLSM can be defined through the action

$$
S=\int d^{D} x\left\{\frac{1}{2} \phi_{a} \square \phi_{a}\right\}
$$

where the fields $\phi_{a}$ are constrained to satisfy $\phi_{a}^{2}=\frac{N}{g}, D$ is the dimension of the space-time and the index $a$ assume the values $1,2, \ldots, N$.

It is useful rewrite the $O(N)$ NLSM action implementing the constraint over $\phi_{a}$ by the use of Lagrange multiplier,

$$
S=\int d^{D} x\left\{\frac{1}{2} \phi_{a} \square \phi_{a}+\sigma\left(\phi_{a}^{2}-\frac{N}{g}\right)\right\}
$$

where the field $\sigma$ is the Lagrange multiplier that constraints $\phi_{a}^{2}=\frac{N}{g}$.

In the late of 1970's the phase structure and the renomalizability of the three-dimensional NLSM was established showing that this model possesses two phases [10, 11]. One phase is $O(N)$ symmetric and exhibits a spontaneous generation of mass due to a non-vanishing vacuum expectation value (VEV) of the Lagrange multiplier field $\sigma$, i.e., $\langle\sigma\rangle \neq 0$. On the other hand, if the fundamental bosonic field $\phi$ acquires a non-vanishing VEV, the $O(N)$ symmetry is spontaneously broken to $O(N-1)$, without any generation of mass. Several extensions of this model was after studied showing no changing in its phase structure [12-19].

The 3D supersymmetric (SUSY) NLSM, in components [14], using the superfield formalism [15], and their noncommutative extensions [16, 17], was shown to be renormalizable to all orders in $1 / N$ expansion. The phase structure of this model was also studied in [18]. In all these papers, a similar conclusion was achieved: no supersymmetry breaking is detected at leading order in $1 / N$ expansion.

The aim of this work is to show that imposing a more general constraint on the SUSY NLSM, the solutions that minimize the effective potential present broken supersymmetry at leading order in the $1 / N$ expansion. Moreover, the $O(N)$ symmetric phase presents a meta-stable vacuum. 


\section{SUPERSYMMETRIC NONLINEAR SIGMA MODEL}

The usual three-dimensional $\mathcal{N}=1$ SUSY NLSM is defined through the action

$$
S=\int d^{5} z\left\{\frac{1}{2} \Phi_{a}(z) D^{2} \Phi_{a}(z)+\Sigma(z)\left[\Phi_{a}(z)^{2}-\frac{N}{g}\right]\right\}
$$

where $\Sigma$ is the Lagrange multiplier superfield that constraints $\Phi_{a}$ to satisfy $\Phi_{a}^{2}(z)=\frac{N}{g}$. With signature $(-,+,+)$, we are using notations and conventions as in 20 . Such definitions and some useful identities can be found in the Supplemental Material [21].

The superfields appearing in this model possess the following $\theta$-expansion:

$$
\begin{aligned}
& \Phi_{a}(x, \theta)=\phi_{a}(x)+\theta^{\beta} \psi_{a \beta}(x)-\theta^{2} F_{a}(x) ; \\
& \Sigma(x, \theta)=\rho(x)+\theta^{\beta} \chi_{\beta}(x)-\theta^{2} \sigma(x) .
\end{aligned}
$$

We can see that the SUSY NLSM possesses more constraints than the non-supersymmetric one. Once the equation of motion of $\Sigma$ constraints

$$
\Phi_{a}^{2}(z)=\left[\phi_{a}^{2}+2 \theta^{\beta} \phi_{a} \psi_{a \beta}-2 \theta^{2}\left(\phi_{a} F_{a}-\frac{1}{2} \psi_{a}^{\beta} \psi_{a \beta}\right)\right]=\frac{N}{g}
$$

it is easy to see that the component fields $\phi_{a}, \psi_{a}^{\alpha}$ and $F_{a}$ must satisfy

$$
\phi_{a}^{2}=\frac{N}{g}, \quad \psi_{a}^{\alpha} \phi_{a}=0, \quad F_{a} \phi_{a}=\frac{1}{2} \psi_{a}^{\beta} \psi_{a \beta}
$$

Beyond the usual constraint $\phi_{a}^{2}=N / g$, the SUSY NLSM also exhibit the constraints $\psi_{a}^{\alpha} \phi_{a}=0$ and $F_{a} \phi_{a}=\frac{1}{2} \psi_{a}^{\beta} \psi_{a \beta}$.

Integrating the Eq. $(3)$ over $d^{2} \theta$, the action of the model can be cast as

$$
\begin{aligned}
S= & \int d^{3} x\left\{\frac{1}{2} \phi_{a} \square \phi_{a}+\frac{1}{2} \psi_{a}^{\alpha} i \partial_{\alpha}{ }^{\beta} \psi_{a \beta}+\frac{1}{2} F_{a}^{2}+\sigma\left(\phi_{a}^{2}-\frac{N}{g}\right)\right. \\
& \left.+2 \rho\left(F_{a} \phi_{a}+\frac{1}{2} \psi_{a}^{\beta} \psi_{a \beta}\right)+2 \chi^{\beta} \psi_{a \beta} \phi_{a}\right\} .
\end{aligned}
$$

Notice that the usual model is obtained setting $\psi=\rho=\chi=0$, and the auxiliary field $\sigma$ must be non-vanishing.

We can eliminate the auxiliary field $F_{a}$ using its equation of motion, $F_{a}=-2 \rho \phi_{a}$. This way, the action

$$
S=\int d^{3} x\left\{\frac{1}{2} \phi_{a} \square \phi_{a}+\frac{1}{2} \psi_{a}^{\alpha} i \partial_{\alpha}{ }^{\beta} \psi_{a \beta}+\sigma\left(\phi_{a}^{2}-\frac{N}{g}\right)-2 \rho^{2} \phi_{a}^{2}+\rho \psi_{a}^{\beta} \psi_{a \beta}+2 \chi^{\beta} \psi_{a \beta} \phi_{a}\right\},
$$


describes the physical content of the model. It is easy to see that if exist a phase where mass is generated to the fundamental fields $\phi$ and $\psi$, their masses will be given by the VEV of the fields $\rho$ and $\sigma$ as

$$
M_{\phi}^{2}=4\langle\rho\rangle^{2}-2\langle\sigma\rangle, \quad M_{\psi}^{2}=4\langle\rho\rangle^{2},
$$

from which we observe that SUSY should be spontaneously broken if $\langle\sigma\rangle \neq 0$, as commented before. For $\langle\sigma\rangle=0$ and for a non-vanishing $\operatorname{VEV}$ of $\rho$, the fundamental bosonic and fermionic fields acquire the same squared mass $4\langle\rho\rangle^{2}$, indicating generation of mass in a supersymmetric phase as is well-known [14-18]. Here we find an intriguing point. While in the non-SUSY model the spontaneous generation of mass occurs due to $\sigma$ acquire a non-vanishing vacuum expectation value, in the SUSY version the field that acts like a "mass generator" to the fundamental fields is $\rho$, which is not present in the non-SUSY model. There is no soft transition or anything that we can interpret as a non-SUSY limit of the spontaneous generation of mass from the SUSY model.

Now, let us define a slightly deformed SUSY NLSM by

$$
S=\int d^{5} z\left\{\frac{1}{2} \Phi_{a}(z) D^{2} \Phi_{a}(z)+\Sigma(z)\left[\Phi_{a}(z)^{2}-\frac{N}{g} \delta(z)\right]\right\}
$$

with the single difference that $\Sigma$ is a Lagrange multiplier superfield that constraints $\Phi_{a}$ to satisfy $\Phi_{a}^{2}(z)=\frac{N}{g} \delta(z)$, where $\delta(z)$ is a constant superfield which possess the $\theta$-expansion $\delta(z)=\delta_{1}-\theta^{2} g \delta_{2}$. Doing $\delta_{2}=0$ and $\delta_{1}=1$ we obtain the usual supersymmetric action for the SUSY NLSM Eq.(3).

The equation of motion of the Lagrange multiplier superfield $\Sigma$ obtained from Eq. (9) generates new constraints to the components of the fundamental superfields $\Phi_{a}$, namely

$$
\phi_{a}^{2}=\frac{N}{g} \delta_{1}, \quad \psi_{a}^{\alpha} \phi_{a}=0, \quad F_{a} \phi_{a}=\frac{1}{2} \psi_{a}^{\beta} \psi_{a \beta}+g \delta_{2} .
$$

To study the phase structure of the model, let us assume that the $\Sigma$ and the N-th component $\Phi_{N}(x, \theta)$ have a constant non-trivial VEV given by

$$
\begin{gathered}
\langle\Sigma\rangle=\Sigma_{c l}=\rho_{c l}-\theta^{2} \sigma_{c l}, \\
\left\langle\Phi_{N}\right\rangle=\sqrt{N} \Phi_{c l}=\sqrt{N}\left(\phi_{c l}-\theta^{2} F_{c l}\right) .
\end{gathered}
$$

Therefore, let us dislocate these superfields by $\Sigma \rightarrow\left(\Sigma+\Sigma_{c l}\right)$ and $\Phi_{N} \rightarrow \sqrt{N}\left(\Phi_{N}+\Phi_{c l}\right)$. So, we can rewrite the action Eq. (3) in terms of the new fields as

$$
\begin{aligned}
S= & \int d^{5} z\left\{\frac{1}{2} \Phi_{a}\left(D^{2}+2 \Sigma_{c l}\right) \Phi_{a}+\Sigma\left(\Phi_{a}^{2}+N \Phi_{c l}^{2}+2 N \Phi_{c l} \Phi_{N}-\frac{N}{g} \delta\right)\right. \\
& \left.+N \Phi_{N}\left(D^{2} \Phi_{c l}+2 \Phi_{c l} \Sigma_{c l}\right)+\frac{N}{2} \Phi_{c l} D^{2} \Phi_{c l}+N \Sigma_{c l}\left(\Phi_{c l}^{2}-\frac{1}{g}\right)\right\} .
\end{aligned}
$$


We can note that the VEV of the superfield $\Sigma, \Sigma_{c l}$, give mass to the fundamental superfields $\Phi_{a}$. This "mass" is $\theta$-dependent, generating different masses to the bosonic and fermionic components of the superfield $\Phi_{a}$, showing a possible phase where supersymmetry is broken.

At leading order, the propagator of $\Phi_{a}$ superfield must satisfy the following equation

$$
\left[D^{2}\left(z_{1}\right)+2 \Sigma_{c l}\right] \Delta\left(z_{1}-z_{2}\right)=i \delta^{(5)}\left(z_{1}-z_{2}\right)
$$

where $\delta^{(5)}\left(z_{1}-z_{2}\right) \equiv \delta^{(3)}\left(x_{1}-x_{2}\right) \delta^{(2)}\left(\theta_{1}-\theta_{2}\right)$, and $\delta^{(2)}(\theta)=-\theta^{2}$.

The solution to the above equation can be obtained from the ansatz

$$
\Delta\left(z_{1}-z_{2}\right)=\left(C_{1}-\theta_{1}^{2} C_{2}-\theta_{2}^{2} C_{3}+\theta_{1}^{\alpha} \theta_{2}^{\beta} \Delta_{\alpha \beta}+\theta_{1}^{2} \theta_{2}^{2} C_{4}\right) \delta^{(3)}\left(x_{1}-x_{2}\right),
$$

where after some algebraic manipulations we can write the propagator of $\Phi_{a}$ superfield as

$$
\Delta(k)=-i \frac{D_{1}^{2}-2 \rho_{c l}}{k^{2}+4 \rho_{c l}^{2}}\left\{1+2 \sigma_{c l} \frac{\delta^{(2)}\left(\theta_{1}\right)\left(D_{1}^{2}+2 \rho_{c l}\right)}{k^{2}+\left(4 \rho_{c l}^{2}-2 \sigma_{c l}\right)}\right\} \delta^{(2)}\left(\theta_{1}-\theta_{2}\right) .
$$

Notice that for $\sigma_{c l}=0$, the above propagator reduces to the usual propagator of a massive scalar superfield. A propagator presenting a similar form was obtained in 22]. See Supplemental Material 21 for details in obtaining the superfield propagator.

From Eq. (12) we can see that exist a mixing between $\Phi_{N}$ and $\Sigma$, but this mixing only contributes to next-to-leading order in $1 / N$ expansion. For now, we can neglect this mixing, since we will deal with the SUSY NLSM at leading order in $1 / N$.

With the propagator of $\Phi_{a}$ superfield, let us evaluate the effective potential through the tadpole method [23 25]. At leading order, the tadpole equation for $\Phi_{N}$ superfield can be cast as

$$
N\left[D^{2} \phi_{c l}+2 \Phi_{c l} \Sigma_{c l}\right]=N\left[F_{c l}+2 \phi_{c l} \rho_{c l}-2 \theta^{2}\left(\phi_{c l} \sigma_{c l}+F_{c l} \rho_{c l}\right)\right]
$$

On the other hand, the tadpole equation for $\Sigma$, Figure 1 , is $\left[N \Phi_{c l}^{2}-\frac{N}{g} \delta+N \int \frac{d^{3} k}{(2 \pi)^{3}} \Delta(k)\right]$. Substituting the expression for $\Delta(k)$, and using the fact that $D^{2} \delta^{(2)}(\theta-\theta)=1$ and $\delta^{(2)}(\theta-\theta)=0$, we obtain

$$
\begin{aligned}
& N \Phi_{c l}^{2}-\frac{N}{g} \delta-i N \int \frac{d^{3} k}{(2 \pi)^{3}}\left\{\frac{1}{k^{2}+\left(4 \rho_{c l}^{2}-2 \sigma_{c l}\right)}+\frac{8 \sigma_{c l} \rho_{c l} \theta^{2}}{\left[k^{2}+\left(4 \rho_{c l}^{2}-2 \sigma_{c l}\right)\right]\left(k^{2}+4 \rho_{c l}^{2}\right)}\right\} \\
= & N\left[\phi_{c l}^{2}-\left(\frac{\delta_{1}}{g}-\frac{1}{g_{c}}\right)-\frac{\sqrt{4 \rho_{c l}^{2}-2 \sigma_{c l}}}{4 \pi}-\theta^{2}\left(2 \phi_{c l} F_{c l}-\frac{2}{\pi} \rho_{c l}^{3 / 2}+\frac{\rho_{c l}}{\pi} \sqrt{4 \rho_{c l}^{2}-2 \sigma_{c l}}+\delta_{2}\right)\right],
\end{aligned}
$$

where $\frac{1}{g_{c}}$ is defined as usual $\int_{\Lambda} \frac{d^{3} k}{(2 \pi)^{3}} \frac{1}{k^{2}}$. The coupling $g_{c}$ is the critical value of $g$ for that the NLSM exhibits the phase transition. 
With the tadpole equations in the hand, the effective potential is obtained integrating Eq.16. over $\Phi_{N}$ and Eq. 17 over $\Sigma$ as

$$
\begin{aligned}
\frac{V_{e f f}}{N} & =-\int d^{2} \theta\left\{\int d \Phi_{N}\left[F_{c l}+2 \phi_{c l} \rho_{c l}-2 \theta^{2}\left(\phi_{c l} \sigma_{c l}+F_{c l} \rho_{c l}\right)\right]\right. \\
& \left.+\int d \Sigma\left[\phi_{c l}^{2}-\lambda-\frac{\sqrt{4 \rho_{c l}^{2}-2 \sigma_{c l}}}{4 \pi}-\theta^{2}\left(2 \phi_{c l} F_{c l}-\frac{2}{\pi} \rho_{c l}\left|\rho_{c l}\right|+\frac{\rho_{c l}}{\pi} \sqrt{4 \rho_{c l}^{2}-2 \sigma_{c l}}+\delta_{2}\right)\right]\right\} \\
& =-\frac{F_{c l}^{2}}{2}-\sigma_{c l}\left(2 \phi_{c l}^{2}-\lambda\right)-6 F_{c l} \rho_{c l} \phi_{c l}+\frac{2}{3 \pi}\left(\rho_{c l}^{2}\right)^{3 / 2}-\frac{4}{3 \pi}\left(\rho_{c l}^{2}-\frac{\sigma_{c l}}{2}\right)^{3 / 2}-\delta_{2} \rho_{c l}+C
\end{aligned}
$$

where $C$ is a constant of integration to be adjusted through the conditions that minimize the effective potential, the gap equations, and $\lambda \equiv\left(\frac{\delta_{1}}{g}-\frac{1}{g_{c}}\right)$ is a parameter that can be positive, negative or zero. In the thermodynamics of NLSM $\lambda$ is interpreted as a quantity proportional to magnetization of the system [13].

Looking to the tadpole equations in Eq.116) and Eq.(17), we observe that the VEV's must to satisfy the following conditions:

$$
\begin{array}{ll}
F_{c l}+2 \phi_{c l} \rho_{c l}=0, & F_{c l} \rho_{c l}+\phi_{c l} \sigma_{c l}=0, \\
\phi_{c l}^{2}-\lambda-\frac{1}{2 \pi} \sqrt{\rho_{c l}^{2}-\frac{\sigma_{c l}}{2}}=0, & \phi_{c l} F_{c l}+\frac{\rho_{c l}}{\pi}\left(\sqrt{\rho_{c l}^{2}-\frac{\sigma_{c l}}{2}}-\left|\rho_{c l}\right|\right)+\frac{\delta_{2}}{2}=0 .
\end{array}
$$

Therefore, setting $C=\left[\sigma_{c l} \phi_{c l}^{2}+4 F_{c l} \rho_{c l} \phi_{c l}+\frac{2}{3 \pi}\left(\rho_{c l}^{2}-\frac{\sigma_{c l}}{2}\right)^{3 / 2}\right]$, the effective potential can be cast as

$$
\frac{V_{e f f}}{N}=-\frac{F_{c l}^{2}}{2}-\sigma_{c l}\left(\phi_{c l}^{2}-\lambda\right)-2 F_{c l} \rho_{c l} \phi_{c l}+\frac{2}{3 \pi}\left(\rho_{c l}^{2}\right)^{3 / 2}-\frac{2}{3 \pi}\left(\rho_{c l}^{2}-\frac{\sigma_{c l}}{2}\right)^{3 / 2}-\delta_{2} \rho_{c l} .
$$

As we did for the classical action, we can eliminate the auxiliary field $F_{c l}$ using its equation of motion,

$$
F_{c l}=-2 \rho_{c l} \phi_{c l}
$$

allowing us to write the effective potential as

$$
\begin{gathered}
\frac{V_{e f f}}{N}=-\sigma_{c l}\left(\phi_{c l}^{2}-\lambda\right)+2 \rho_{c l}^{2} \phi_{c l}^{2}+\frac{2}{3 \pi}\left[\left(\rho_{c l}^{2}\right)^{3 / 2}-\left(\rho_{c l}^{2}-\frac{\sigma_{c l}}{2}\right)^{3 / 2}\right]-\delta_{2} \rho_{c l} . \\
-N \Phi_{c l}^{2}+\frac{N}{g}=0
\end{gathered}
$$

Figure 1. Tadpole equation of $\Sigma$ at leading order. Continuous lines represent the $\Phi_{a}$ superfield propagator, while cut dashed line a removed external $\Sigma$ propagator. 
From the effective potential Eq. 22 , the conditions that extremize the effective potential are given by

$$
\begin{aligned}
& \phi_{c l}\left(\rho_{c l}^{2}-\frac{\sigma_{c l}}{2}\right)=0, \\
& \phi_{c l}^{2}-\lambda-\frac{1}{2 \pi} \sqrt{\rho_{c l}^{2}-\frac{\sigma_{c l}}{2}}=0, \\
& \rho_{c l}\left(2 \pi \phi_{c l}^{2}+\left|\rho_{c l}\right|-\sqrt{\rho_{c l}^{2}-\frac{\sigma_{c l}}{2}}\right)=\frac{\pi}{2} \delta_{2} .
\end{aligned}
$$

Solving these equations, we determine the field configurations that extremize the effective potential. Such solutions are presented in two phases, one $O(N)$ symmetric phase and another $O(N)$ broken to $O(N-1)$. The $O(N)$ symmetric phase, $\lambda<0$ or $g>g_{c}$, the solutions are given by:

$$
\begin{aligned}
& \phi_{c l}=0, \quad \rho_{c l}=\pi|\lambda|+\frac{1}{2} \sqrt{2 \pi\left(2 \pi \lambda^{2}-\delta_{2}\right)}, \quad \sigma_{c l}=\frac{1}{2}\left[2 \pi|\lambda|+\sqrt{2 \pi\left(2 \pi \lambda^{2}-\delta_{2}\right)}\right]^{2}-8 \pi^{2} \lambda^{2} \\
& \phi_{c l}=0, \quad \rho_{c l}=-\pi|\lambda|-\frac{1}{2} \sqrt{2 \pi\left(2 \pi \lambda^{2}+\delta_{2}\right)}, \quad \sigma_{c l}=\frac{1}{2}\left[2 \pi|\lambda|+\sqrt{2 \pi\left(2 \pi \lambda^{2}+\delta_{2}\right)}\right]^{2}-8 \pi^{2} \lambda^{2}
\end{aligned}
$$

Note for real solutions, the parameter $\delta_{2}$ is constrained to be $\left|\delta_{2}\right| \leq 2 \pi \lambda^{2}$. Moreover, as we will see, exist a $\delta_{2} \neq 0$ which $V_{\text {eff }}$ assumes its minimum value. Setting $\delta_{2}=0$ we have the well-known solutions [14 18]

$$
\rho_{c l}= \pm 2 \pi|\lambda|, \quad \phi_{c l}=F_{c l}=\sigma_{c l}=0 .
$$

The solution Eq. (24) is the global minimum of the effective potential while Eq. (25) is a local one. The effective potential is plotted in the Figure 2 as a function of $\rho_{c l}$ and $\phi_{c l}$, where it is possible to see the true and the false vacua.
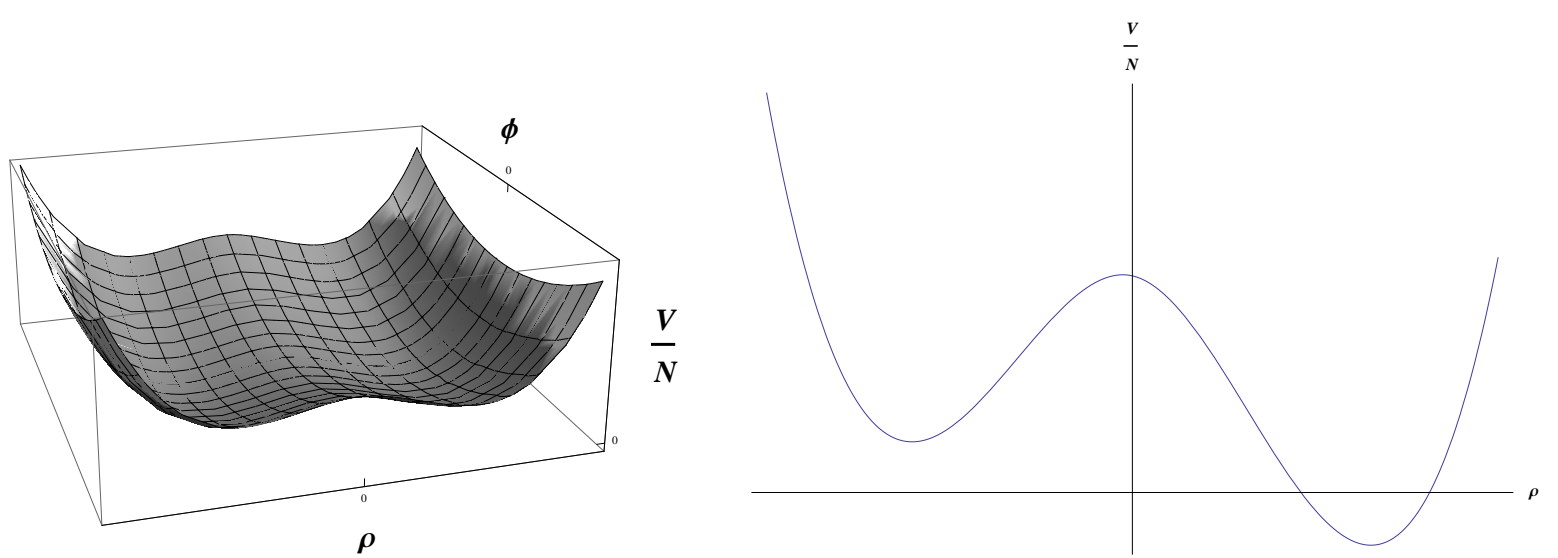

Figure 2. Effective potential in the $O(N)$ symmetric phase as function of $\rho_{c l}$ and $\phi_{c l}$. The plot in the right side of the figure is a slice of the $V_{e f f}$ at $\phi_{c l}=0$, evidencing the presence of a meta-stable vacuum. 
In the minimum, $V_{\text {eff }}$ is negative, this is because we are dealing with an explicit breaking of supersymmetry. The generated masses for the fundamental fields $\phi$ and $\psi$ in the $O(N)$ symmetric phase are given by

$$
\begin{aligned}
& M_{\phi}^{2}=4\langle\rho\rangle^{2}-2\langle\sigma\rangle=16 \pi^{2} \lambda^{2} \\
& M_{\psi}^{2}=4\langle\rho\rangle^{2}=8 \pi^{2} \lambda^{2}+4 \pi|\lambda| \sqrt{2 \pi\left(2 \pi \lambda^{2}-\delta_{2}\right)}-2 \pi \delta_{2} .
\end{aligned}
$$

In the limit $\delta_{2} \rightarrow 0$ the masses $M_{\phi}^{2}=M_{\psi}^{2}$ and supersymmetry is restored.

The second phase, $O(N)$ symmetry is broken to $O(N-1), \lambda>0$ or $g<g_{c}$, and the solutions that minimize the effective potential are given by

$$
\begin{array}{ll}
\phi_{c l}= \pm \sqrt{\lambda}, & \rho_{c l}=\pi \lambda-\frac{1}{2} \sqrt{2 \pi\left(2 \pi \lambda^{2}-\delta_{2}\right)}, \quad \sigma_{c l}=\frac{1}{2}\left[2 \pi \lambda-\sqrt{2 \pi\left(2 \pi \lambda^{2}-\delta_{2}\right)}\right]^{2} ; \\
\phi_{c l}= \pm \sqrt{\lambda}, & \rho_{c l}=-\pi \lambda+\frac{1}{2} \sqrt{2 \pi\left(2 \pi \lambda^{2}+\delta_{2}\right)}, \quad \sigma_{c l}=\frac{1}{2}\left[2 \pi \lambda-\sqrt{2 \pi\left(2 \pi \lambda^{2}+\delta_{2}\right)}\right]^{2} .
\end{array}
$$

where, just as $O(N)$ symmetric phase discussed before, for $\delta_{2} \rightarrow 0$ the above solutions collapse to

$$
\phi_{c l}= \pm \sqrt{\lambda}, \quad F_{c l}=\sigma_{c l}=\rho_{c l}=0 .
$$

Just as the supersymmetric and non-supersymmetric cases, in the $O(N)$ symmetric phase the scalar field $\phi$ is kept massless, i.e., $M_{\phi}^{2}=0$. But, due to the parameter that breaks supersymmetry, $\delta_{2}$, the fundamental fermion of the model acquires the mass

$$
M_{\psi}^{2}=4\langle\rho\rangle^{2}=\left[2 \pi \lambda-\sqrt{2 \pi\left(2 \pi \lambda^{2}-\delta_{2}\right)}\right]^{2} .
$$

It is easy to see that if $\delta_{2} \rightarrow 0$ so $M_{\psi}^{2} \rightarrow 0$.

Finally, let us deal with the optimal value of the SUSY-breaking parameter $\delta_{2}$. Eliminating, from Eq. 22 , all fields by the use of their equations of motion, except the fundamental field $\phi$, to $\lambda>0$ we find

$$
\begin{aligned}
\frac{V_{e f f}}{N}= & \frac{1}{6}\left\{-12 \pi \lambda\left(\delta_{2}+2 \pi \lambda^{2}\right)-3\left(4 \pi \lambda^{2}-\delta_{2}\right) \sqrt{2 \pi\left(2 \pi \lambda^{2}-\delta_{2}\right)}\right. \\
& +\left[32 \pi^{4} \lambda^{2}-8 \pi^{3} \delta_{2}-16 \pi^{3} \lambda \sqrt{2 \pi\left(2 \pi \lambda^{2}-\delta_{2}\right)}\right]^{3 / 2} \\
& \left.+144 \pi^{2} \lambda \phi_{c l}^{2}\left(\lambda-\phi_{c l}^{2}\right)+48 \pi^{2} \phi_{c l}^{6}-32 \pi^{2}\left|\lambda-\phi_{c l}^{2}\right|\right\} .
\end{aligned}
$$

Minimizing Eq. (33) for $\delta_{2}$ we obtain the solution

$$
\delta_{2}=\frac{3 \pi}{2} \lambda^{2} .
$$

The effective potential Eq. 222 evaluated for $\delta_{2}=\frac{3 \pi}{2} \lambda^{2}$ is given by

$$
\frac{V_{e f f}}{N}=-\sigma_{c l}\left(\phi_{c l}^{2}-\lambda\right)+2 \rho_{c l}^{2} \phi_{c l}^{2}+\frac{2}{3 \pi}\left[\left(\rho_{c l}^{2}\right)^{3 / 2}-\left(\rho_{c l}^{2}-\frac{\sigma_{c l}}{2}\right)^{3 / 2}\right]-\frac{3 \pi}{2} \lambda^{2} \rho_{c l} .
$$


One interesting note is that $\delta_{2}=0$ becomes a local maximum in this model. Once introduced the SUSY-breaking parameter, the supersymmetric solutions are not the solutions that minimize the effective potential anymore.

\section{FINAL REMARKS}

Summarizing, the three-dimensional supersymmetric nonlinear sigma model, deformed by a nonsupersymmetric constraint, possess two phases. In the first one is the $O(N)$ symmetric phase, $\lambda<0$ or $g>g_{c}$, which possess the remarkable characteristic of the presence of a meta-stable vacuum. In this phase, all fields acquire a non-vanishing vacuum expectation value, generating masses to the fundamental fields $\phi$ and $\psi$. These masses are different for non-vanishing $\delta_{2}$, coupling responsible for supersymmetry breaking. In the limit $\delta_{2} \rightarrow 0$ the masses of $\phi$ and $\psi$ tend to be equal, restoring the supersymmetry. In the $O(N)$ broken phase, only the components of the Lagrange multiplier superfield acquire a non-vanishing vacuum expectation value, generating mass to the fermionic field $\psi$ and keeping $\phi$ massless. Also in this phase, the limit $\delta_{2} \rightarrow 0$ can be taken to restore the supersymmetric solutions. An important note is the fact that $\delta_{2}$ can not be chosen arbitrarily. It possesses an optimal value that minimizes the effective potential.

Finally, we think that gauge and noncommutative extensions (with constant noncommutative parameter; see, for example the SUSY CP ${ }^{(N-1)}$ model presented in Ref. [26]) of this model should present similar structure, including the presence of the meta-stable vacuum, since in general the tadpole diagrams in noncommutative models are the same of the commutative ones.

Acknowledgments. This work was partially supported by the Brazilian agencies Fundação de Amparo à Pesquisa do Estado de São Paulo (FAPESP), Conselho Nacional de Desenvolvimento Científico e Tecnológico (CNPq) and Fundação de Apoio à Pesquisa do Rio Grande do Norte (FAPERN).

[1] M. Gell-Mann and M. Levy, Nuovo Cim. 16, 705 (1960).

[2] A. M. Polyakov, Phys. Lett. B 59, 79 (1975).

[3] E. Brezin and J. Zinn-Justin, Phys. Rev. Lett. 36, 691 (1976).

[4] E. Brezin and J. Zinn-Justin, Phys. Rev. B 14, 3110 (1976).

[5] W. A. Bardeen, B. W. Lee and R. E. Shrock, Phys. Rev. D 14, 985 (1976).

[6] D. Friedan, Phys. Rev. Lett. 45, 1057 (1980). 
[7] S. Hikami, Phys. Lett. B 98, 208 (1981).

[8] L. F. Alday and J. M. Maldacena, JHEP 0711, 019 (2007).

[9] Z. Bajnok, J. Balog, B. Basso, G. P. Korchemsky and L. Palla, Nucl. Phys. B 811, 438 (2009).

[10] I. Y. Arefeva, Annals Phys. 117, 393 (1979).

[11] I. Y. Arefeva, E. R. Nissimov and S. J. Pacheva, Commun. Math. Phys. 71, 213 (1980).

[12] I. Y. Arefeva and S. I. Azakov, Nucl. Phys. B 162, 298 (1980).

[13] B. Rosenstein, B. J. Warr and S. H. Park, Nucl. Phys. B 336, 435 (1990).

[14] V. G. Koures and K. T. Mahanthappa, Phys. Rev. D 43, 3428 (1991) [Erratum-ibid. D 45, 717 (1992)].

[15] V. G. Koures and K. T. Mahanthappa, Phys. Rev. D 45, 580 (1992).

[16] H. O. Girotti, M. Gomes, V. O. Rivelles and A. J. da Silva, Int. J. Mod. Phys. A 17, 1503 (2002).

[17] H. O. Girotti, M. Gomes, A. Y. Petrov, V. O. Rivelles and A. J. da Silva, Phys. Lett. B 521, 119 (2001).

[18] T. Matsuda, J. Phys. G 22, L119 (1996).

[19] I. Jack, D. R. T. Jones and N. Mohammedi, Phys. Lett. B 520, 405 (2001).

[20] S. J. Gates, M. T. Grisaru, M. Rocek and W. Siegel, Front. Phys. 58, 1 (1983).

[21] See Supplemental Material at http://link.aps.org/supplemental/10.1103/PhysRevD.88.067702 for details in obtaining the supereld propagator.

[22] J. L. Boldo, L. P. Colatto, M. A. De Andrade, O. M. Del Cima and J. A. Helayel-Neto, Phys. Lett. B 468, 96 (1999).

[23] S. Weinberg, Phys. Rev. D 7, 2887 (1973).

[24] R. D. C. Miller, Nucl. Phys. B 228, 316 (1983).

[25] R. D. C. Miller, Nucl. Phys. B 229, 189 (1983).

[26] A. F. Ferrari, A. C. Lehum, A. J. da Silva and F. Teixeira, J. Phys. A 40, 7803 (2007). 